\title{
Vesicular Glutamate Transporter 2 Is Associated with the Cochlear Nucleus Commissural Pathway
}

\author{
Jianxun Zhou ${ }^{1,3}$, Chunhua Zeng ${ }^{1}$, Yilei Cui ${ }^{1}$, and Susan Shore ${ }^{1,2}$ \\ ${ }^{1}$ Kresge Hearing Research Institute, Department of Otolaryngology, University of Michigan, 1150 W. Medical Center Drive, \\ Room 5434A Med Sci I, Ann Arbor, MI 48109-5616, USA \\ ${ }^{2}$ Department of Molecular and Integrative Physiology, University of Michigan, Ann Arbor, MI 48109, USA \\ ${ }^{3}$ Present address: Department of Physical Medicine Eै Rehabilitation, Albany Medical Center, Albany, NY 12208, USA
}

Received: 25 February 2010; Accepted: 1 June 2010; Online publication: 24 June 2010

\begin{abstract}
The cochlear nucleus $(\mathrm{CN})$ is the first auditory structure to receive binaural information via $\mathrm{CN}$-commissural connections. In spite of an abundance of evidence that CN-commissural neurons are glycinergic and thus inhibitory, physiological, and anatomical evidence suggests that a small group of $\mathrm{CN}$-commissural neurons are excitatory. In this study, we examined the excitatory portion of the $\mathrm{CN}$-commissural pathway by combining anterograde tract tracing with immunohistochemistry of vesicular glutamate transporters (VGLUTs) and retrograde tract tracing with immunohistochemistry of glycine and GABA. VGLUTs accumulate glutamate in synaptic vesicles and are prime markers for glutamatergic neurons. The terminal endings of $\mathrm{CN}$-commissural projections were typically en passant or small terminal boutons, but large, irregular swellings were also observed, confined to the granule cell domain (GCD). Both small and large terminal endings in the GCD colabeled with VGLUT2, but not VGLUT1. In addition, some CN-commissural cells themselves received VGLUT2-positive puncta on their somata. After large injections into the $\mathrm{CN}, 37 \%$ of the total number of retrogradely labeled commissural neurons was immunonegative to glycine or GABA. Retrograde labeling after a restricted GCD injection revealed a majority of putative excitatory CN-commissural neurons as multipolar, in the marginal regions of the ventral $\mathrm{CN}$,
\end{abstract}

Correspondence to: Susan Shore - Kresge Hearing Research Institute, Department of Otolaryngology · University of Michigan $\cdot 1150 \mathrm{~W}$. Medical Center Drive, Room 5434A Med Sci I, Ann Arbor, MI 48109-5616, USA. Telephone: +1-734-6472116; fax: +1-734-7640014; email: sushore@umich.edu medially as well as in the small cell cap region and deep dorsal CN. These results provide direct anatomical evidence that an excitatory commissural projection is present, and VGLUT2 is associated with this pathway both as its source and as a recipient.

Keywords: excitatory, glutamatergic, binaural, tract tracing, immunohistochemistry

\section{INTRODUCTION}

The cochlear nucleus $(\mathrm{CN})$ is the first auditory structure to receive binaural information directly via the $\mathrm{CN}$ commissural pathway. $\mathrm{CN}$-commissural projections terminate in most regions of the $\mathrm{CN}$ including the granule cell domain (GCD), which includes the external shell of the ventral CN (VCN) and the fusiform cell layer of the dorsal CN (DCN) (Schofield and Cant 1996; Weedman et al. 1996; Zhou et al. 2007). A common assumption over the past two decades has been that the majority of $\mathrm{CN}$-commissural projections arise from the broadly tuned, inhibitory, D stellate or type II multipolar cells (Cant and Benson 2003; Doucet and Ryugo 2006; Smith et al. 2005; Smith and Rhode 1989). However, recent observations (Doucet et al. 2009) suggest that this pathway is composed of at least two populations of cells: large multipolar cells that resemble the glycinergic, D stellate cells, as well as small- and medium-sized multipolar cells that account for approximately $60 \%$ of $\mathrm{CN}$ commissural neurons. These cells have type I axosomatic innervation (i.e., receive few terminals on their somata) and are located in the marginal region of VCN as well as 
other regions of the VCN. Cumulative evidence suggests that some $\mathrm{CN}$-commissural neurons are excitatory (Alibardi 1998; Babalian et al. 2002; Shore et al. 1992). Alibardi $(1998,2000,2006)$ reported that about $60 \%$ of CN-commissural neurons are immunonegative to glycine or GABA. Furthermore, electron microscopic examination revealed that most large $\mathrm{CN}$-commissural terminals in the GCD contained round vesicles and formed asymmetric synapses, suggestive of excitatory synapses (Alibardi 2000, 2006). In contrast, the majority of CN-commissural terminal boutons in the central regions of VCN contained pleomorphic vesicles and formed symmetric synaptic thickenings. These terminal endings primarily colabeled with glycine (Alibardi 2000).

Physiological studies have shown that a small percentage $(2-4 \%)$ of units in the VCN showed excitatory responses to contralateral acoustic stimulation (Shore et al. 2003; Sumner et al. 2005), in addition to the commonly recorded inhibitory responses (Babalian et al. 2002; Ingham et al. 2006; Needham and Paolini 2003, 2007; Shore et al. 2003; Sumner et al. 2005). Although contralateral excitation is rare in normalhearing animals, it can increase significantly after ipsilateral conductive (up to $30 \%$ ) or sensorineural $(>60 \%$ ) hearing loss (Bledsoe et al. 2009; Sumner et al. 2005). These studies question previous assumptions that the CN-commissural pathway is exclusively inhibitory and what the source of excitatory commissural neurons might be.

We examined the putative excitatory portion of the CN-commissural pathway using anterograde tract tracing combined with immunolabeling of vesicular glutamate transporters (VGLUTs). VGLUTs accumulate glutamate in synaptic vesicles and are prime markers for excitatory glutamatergic terminals (Fremeau et al. 2002, 2001; Gabellec et al. 2007; Kaneko et al. 2002; Zhou et al. 2007). In addition, we conducted retrograde tract tracing experiments combined with immunohistochemical identification of glycine and GABA to identify which $\mathrm{CN}$-commissural neurons were not inhibitory.

\section{METHODS}

\section{Animal preparations and surgical procedures}

The experiments were performed on 11 pigmented guinea pigs with normal Preyer's reflexes, weighing 300-400 g. All procedures were performed in accordance with the NIH guidelines for the use and care of laboratory animals (NIH publication no. 80-23) and the guidelines provided by the University Committee on Use and Care of Animals of University of Michigan.

Animals were anesthetized with intramuscular injections of ketamine hydrochloride (Ketaset; $40 \mathrm{mg} / \mathrm{kg}$ ) and xylazine (Rompun; $10 \mathrm{mg} / \mathrm{kg}$ ). Rectal temperature was maintained at $38 \pm 0.5^{\circ} \mathrm{C}$ with a thermostati- cally controlled heating pad. The left $\mathrm{CN}$ was exposed by aspirating part of the cerebellum. In the retrograde tracing experiments $(n=5)$, a total volume of $0.1-$ $0.4 \mu \mathrm{l}$ tracers [2\% Fluorogold (FG, Fluorochrome, Englewood, $\mathrm{CO} ; n=4)$ or $10 \%$ biotinylated dextranamine (BDA, MW 10,000, Molecular Probes; in phosphate-buffered saline, or PBS; $n=1$ )] was pressureinjected into one $\mathrm{CN}$ using a Hamilton microsyringe equipped with a glass micropipette $(20-30 \mu \mathrm{m}$ tip). In the anterograde tracing experiments $(n=6)$, a total volume of $0.1-0.4 \mu \mathrm{l}$ of BDA was pressure-injected into the left CN. Previous studies and our concurrent retrograde experiments indicate that cells at the intersection of DCN and VCN are more likely to be excitatory (i.e., glycine and GABA negative, Alibardi 2006) and project to the contralateral GCD. Thus, attempts were made specifically to deposit anterograde tracers in the medial edge region of the VCN, near to its intersection with DCN. Retrograde tracers were placed in the entire $\mathrm{CN}$, but some were restricted to the shell region.

Four to six days after the injections, animals were euthanized with Nembutal (15 mg/kg, i.p.) and transcardially perfused with $0.1 \mathrm{M}$ PBS, followed by fresh $4 \%$ paraformaldehyde in PBS used within $1 \mathrm{~h}$ of preparation (Pow et al. 1995). One animal was euthanized 1 day after injection. Fluorogold can retrogradely label somata with wide range of survival times, ranging from 1-2 days to 2 months (Schmued and Fallon 1986). This animal still showed sufficient labeling and therefore was included in the study. Following perfusion-fixation, the brain was isolated and placed in the same fixative for $2 \mathrm{~h}$ at $4^{\circ} \mathrm{C}$. The brain was transferred into $20 \%$ sucrose in $0.1 \mathrm{M}$ PBS overnight at $4^{\circ} \mathrm{C}$. The brainstem was then sectioned on a freezing microtome at a thickness of $40 \mu \mathrm{m}$.

\section{Tissue processing and immunocytochemistry}

Retrograde tracing experiments. Unless stated otherwise, tissue processing and immunocytochemistry were performed at $4^{\circ} \mathrm{C}$ using the following protocol. Floating sections were incubated in blocking solutions containing $1 \%$ normal goat serum (Jackson ImmunoResearch Laboratories, 005-000-121) in 0.1 M phosphate buffer with $0.1 \%$ Triton X-100 (Sigma 900293-1), pH 7.4. The same blocking solution was also used to dilute primary and secondary antibodies. After 30-minute incubation in blocking solution, floating sections were treated overnight with primary antibodies (polyclonal rat antiglycine antibody 1:1000, Cat\# IG1002, ImmunoSolution, Australia; polyclonal rabbit anti-GABA antibody 1:200, Cat\# A2052, Sigma St. Louis, MO) to determine if retrogradely labeled cells were glycinergic or GABAergic. Sections were thoroughly washed in PBS and incubated with 
secondary antibodies [Cy3 conjugated goat anti-rat 1:300 (Molecular Probes) and Cy2 conjugate goat anti-rabbit 1:300 (Molecular Probes)] for $2 \mathrm{~h}$. After washing in PBS three times, sections were mounted on clean slides, dehydrated in graded ethanol, cleared in Micro-clear (Micron, Fairfax, VA) and cover-slipped with Micro-cover (Micron, Fairfax, VA).

Both anti-glycine and anti-GABA antibodies detect the corresponding amino acids very well in paraformaldehyde-fixed tissues (Allain et al. 2006; Crook et al. 2006; Ohshita et al. 2004; Pow et al. 1995; Xin et al. 2007). Polyclonal rat antiglycine antibody was generated in rats against glycine that had been conjugated to a carrier protein [bovine serum albumin (BSA)] using formaldehyde. Sensitivity and specificity of this antibody in different species have been previously verified (Pow et al. 1995). Since cells in superficial DCN (presumably cartwheel cells or stellate cells) express both GABA and glycine, and cells in inferior colliculus primarily express GABA (Gleich and Vater 1998; Kolston et al. 1992), we used DCN superficial cells and cells in inferior colliculus as the positive reference control. Negative controls were conducted on sections that were not treated with either primary or secondary antibodies, resulting in no immunolabeling. Preincubation of the glycine antibody with glycineformaldehyde-BSA (Cat\#GLYF01, ImmunoSolution, Australia) resulted in negative immunolabeling. Although the immunogen used for production of polyclonal rabbit GABA antibody is BSA conjugated to GABA, this antibody also works well with paraformadehyde-fixed tissues (Allain et al. 2006; Crook et al. 2006; Ohshita et al. 2004; Xin et al. 2007). Nonetheless, we still performed a preadsorption control study by preincubation of the GABA antibody with GABAglutaraldehyde-BSA (Cat\# GABAG01, ImmunoSolution, Australia), which resulted in negative immunolabeling (Fig. 1).

Some tissues from these animals with FG injections were saved for VGLUT immunoreactivity studies to evaluate if $\mathrm{CN}$-commissural neurons receive VGLUT2 mediated synaptic inputs (see below, "Anterograde Tracing Experiments").

For one animal, the labeled $\mathrm{CN}$-commissural neurons and their colabeling with GABA or glycine were manually counted on every fourth section. Retrogradely labeled neurons were mapped onto templates that included six transverse sections evenly extending from the caudal to rostral ends of the $\mathrm{CN}$ (i.e., the 14.3th, 28.6th, 42.9th, 57.2th, 71.5th, 85.8th percentiles). The number of terminal counts was multiplied by 4 to achieve the total terminal counts and corrected for double counting errors using Abercrombie's correction [corrected number $=$ count $\times$ (section thickness)/(section thickness + terminal size)] (Abercrombie 1946).
Another animal in this group that received an injection of $\mathrm{BDA}$ in the $\mathrm{CN}$ was used to document the CN-commissural neurons that specifically project to contralateral shell regions. Tissues from this animal were processed differently: brain sections were directly mounted on clean glass slides and air dried. Sections were treated for 30 minutes in $3 \%$ hydrogen dioxide in methanol, followed by a 2-h incubation with avidinbiotinylated horseradish peroxidase (Vectastain Elite ABC kit, Vector Laboratories, Inc., Burlingame, CA), then reacted with 3,31-diaminobenzidine tetrahydrochloride (DAB, Vector Laboratories, Inc., Burlingame, CA). Slides were counterstained with neutral red, dehydrated, cover-slipped, and examined using a lightfield microscope (Leica DML).

Anterograde tracing experiments. After incubating with blocking solution, alternate floating sections were incubated overnight with primary antibodies to VGLUT1 (1:1000, Synaptic Systems, Germany, Cat.\# 135 303) and VGLUT2 (1:1000, Synaptic Systems, Germany, Cat.\# 135 403), respectively. Following thorough washing in PBS, sections were reacted for $2 \mathrm{~h}$ with secondary antibodies containing Cy3conjugated goat anti-rabbit (Molecular Probes) and Alexa-488-conjugated streptavidin (1:300, to visualize BDA-labeled terminals). Sections were then processed according the procedures described above.

\section{Image processing and confocal laser microscopy}

Sections were examined using a fluorescent microscope equipped with the appropriate filters (Leica, DM). Photomicrographs of immunolabeling were digitized and imported to Adobe Photoshop for final contrast adjustment. Colocalizations were also examined using confocal laser microscopy (Zeiss LSM 510). Images immunolabeled with Cy2 and Cy3 were scanned with lasers of Argon and HeNe1, respectively. Images immunolabeled with FG were scanned with a UV laser with an excitation wavelength of $364 \mathrm{~nm}$, filtered using an emission LP filter at $560 \mathrm{~nm}$, and viewed on the blue channel. Confocal images were acquired at a $1-\mu \mathrm{m}$ optical thickness. To obtain a clear view of terminal or somata profiles, a series of 3-12 serial optical sections were obtained to generate a Z-projection of stacks. Puncta or somata that were yellow on the confocal multichannel images indicated colocalization. Photomicrographs of labeled neurons and terminals were digitized and imported to Adobe Photoshop for labeling and contrast adjustment.

We used a fluorescent microscope equipped with the appropriate filters (Leica, DM) to examine the $\mathrm{CN}$ commissural neurons that colabeled with glycine or GABA. The difference between a neuronal soma and a terminal ending can be determined by visualizing a 

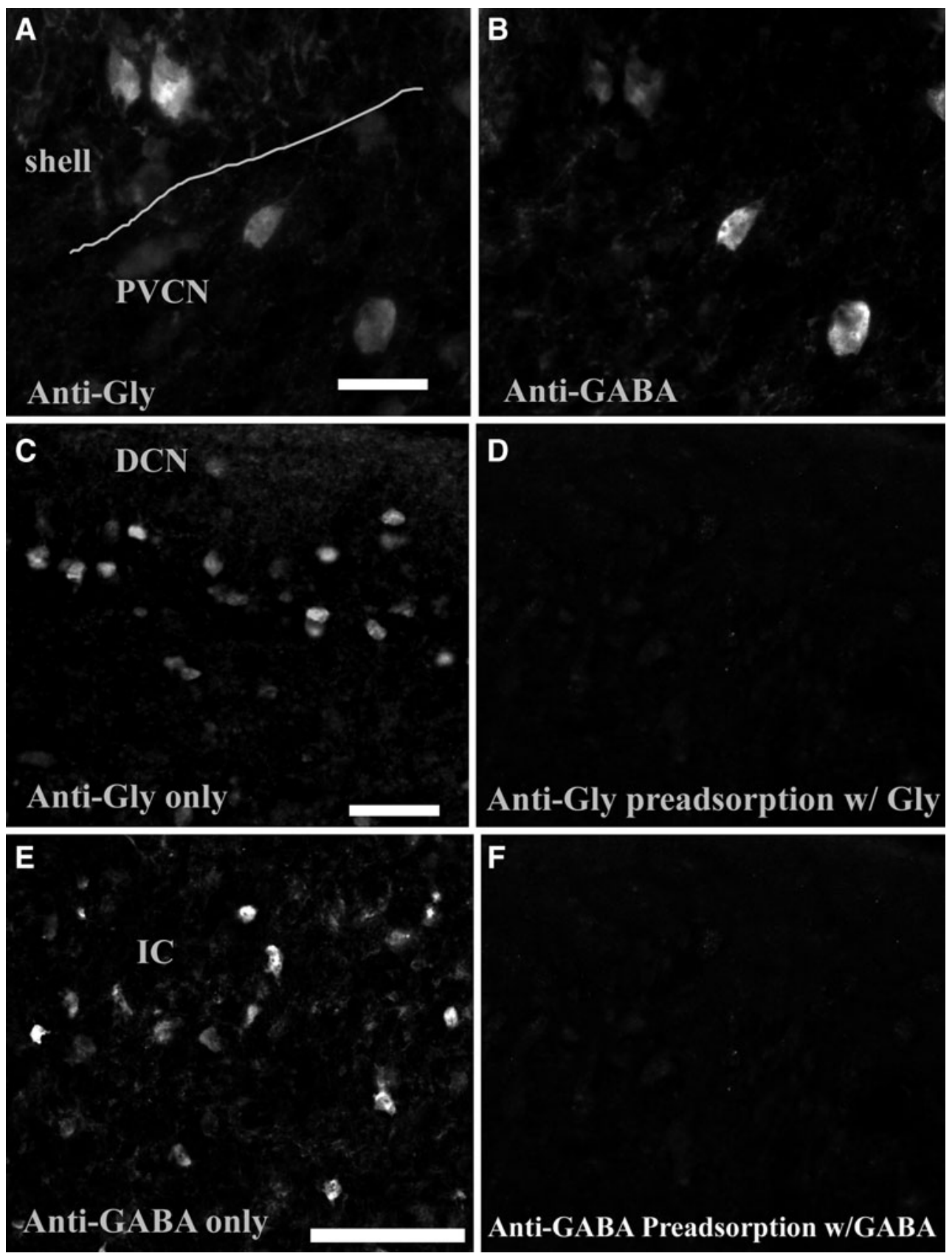

FIG. 1. Immunoreactivity of glycine and GABA in the $\mathrm{CN}$. A and $\mathbf{B}$ Photomicrographs of glycine and GABA immunoreactivity in the VCN (40×). Both photomicrographs (A and B) are taken from the same $\mathrm{CN}$ region. The line in $\mathbf{A}$ indicates the boundary between the shell region and central PVCN. The two large neurons shown in central VCN are clearly colabeled with both glycine (A) and GABA (B). The two shell neurons are also colabeled but very lightly for GABA. C

central clearing with adjustment of the focus of the objective lens. Double-labeling of CN-commissural neurons with glycine and/or GABA was determined by frequently switching the filters and adjusting the focus of the objective lens. Colocalization was established when the two different labels exactly marked the same profile at the same focusing level. This method of identifying

Photomicrographs of glycine immunoreactivity in DCN layer 2 (20x). Cells show positive glycine immunoreactivity and likely are cartwheel cells. D Preadsorbtion of antiglycine antibody with glycine-BSA resulted in negative immunoreactivity in DCN layer 2. E Photomicrographs of immunoreactivity of GABA in the IC $(20 \times)$. F Preadsorbtion of anti-GABA antibody with GABA-BSA resulted in negative immunoreactivity in IC. Scale bars in $\mathbf{A}, \mathbf{C}, \mathbf{E}=50 \mu \mathrm{m}$.

double-labeled CN commissural terminals agreed well with the results from confocal images. Additionally, we used a strict criterion for selecting glycine-negative and/or GABA-negative CN commissural neuronsonly those that were unequivocally immunonegative to glycine and GABA were selected. Cells that showed weak or questionable glycine or GABA immunoreac- 
tivity were still considered as positive and classified as double-labeled CN commissural neurons. This approach yielded increased specificity of glycine/GABA-negative CN commissural neurons.

Labeled CN-commissural neurons were further classified as (1) medium-to-large cells with the long axis greater than $16 \mu \mathrm{m}$ and (2) small cells had a long axis smaller than or equal to $16 \mu \mathrm{m}$ (Shore et al. 1992). This resulted in eight categories according to size and immunoreactivity to glycine and GABA antibodies: (1-4) medium-to-large CN commissural neurons: glycine positive and GABA positive ( $\mathrm{Gly}+/ \mathrm{GABA}+)$, glycine positive and GABA negative (Gly+/GABA-), glycine negative and GABA positive (Gly-/GABA+), glycine negative and GABA negative (Gly-/GABA-); (5-8) small CN commissural cells: same four categories as medium-to-large cells. These retrogradely labeled neurons were mapped onto templates that included six transverse sections evenly extending from the caudal to rostral ends of the CN (i.e., the 14.3th, 28.6th, 42.9th, 57.2 th, 71.5th, 85.8th percentiles). In cases where the $\mathrm{BDA}$ reaction product or $\mathrm{FG}$ filled both the soma and dendrites of a labeled cell, we tried to identify the cell type using the scheme of Hackney et al. (1990).

\section{RESULTS}

\section{CN-commissural terminals colabel with VGLUT2}

Following injections of BDA in the left $\mathrm{CN}$, labeled fibers entered the right $\mathrm{CN}$ through either the dorsal/intermediate acoustic striae or the trapezoid body. Labeled fibers and puncta were found in most regions of the $\mathrm{CN}$. The labeled puncta were usually of small to medium size $(1-2.5 \mu \mathrm{m})$, but a few puncta were large and irregular $(>2.5 \mu \mathrm{m})$. These large puncta were found exclusively in the GCD. Many large, irregular puncta (Fig. 2, arrows) and some small
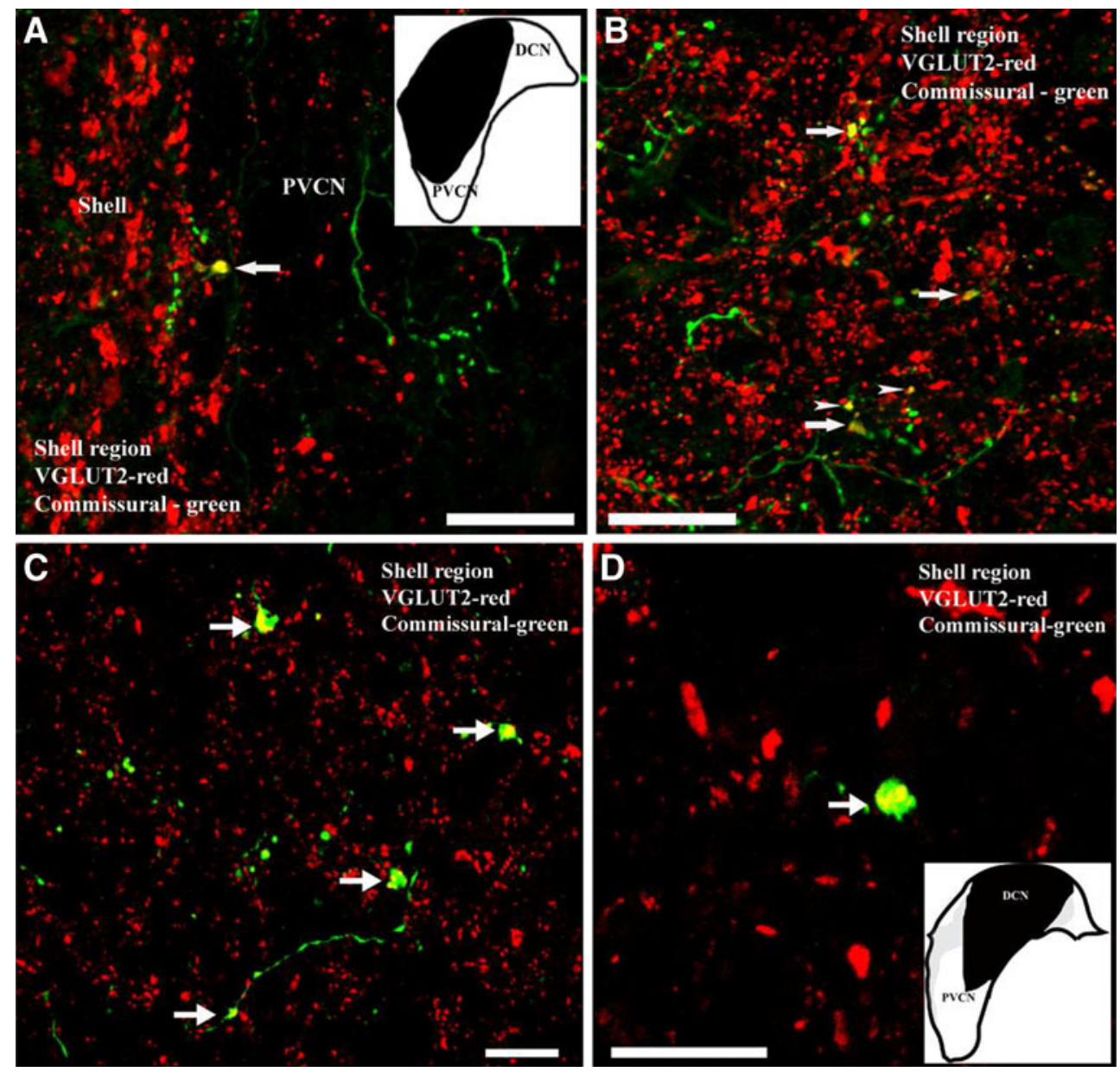

FIG. 2. Confocal images showing colocalization of anterogradely labeled $\mathrm{CN}$-commissural terminal endings with VGLUT2 immunoreactivity in the shell region. Image A and B $(40 \times)$ are from one animal with injection site shown in inset of $\mathbf{A}$; image $\mathbf{C}(60 \times)$ and $\mathbf{D}$ (100X) are from another animal with injection site shown in inset of D. A, B Large (arrows) and small (arrowheads) CN-commissural

terminal endings colabeled with VGLUT2 immunoreactivity in the shell region. C, D CN-commissural terminals in shell region (arrow), but not in magnocellular region of VCN, colabeled with VGLUT2 immunoreactivity. Red, VGLUT2 immunoreactivity; green, CNcommissural labeling; yellow, double-labeled terminals. Scale bars= $50 \mu \mathrm{m}(\mathbf{A}$ and $\mathbf{B})$ and $20 \mu \mathrm{m}(\mathbf{C}$ and $\mathbf{D})$. 
puncta (Fig. 2, arrowheads) in the GCD colabeled with VGLUT2. None of the CN commissural puncta in any region colabeled with VGLUT1 (data not shown).

$\mathrm{CN}$-commissural cells that are immunonegative for glycine and GABA mostly are located in marginal regions to the $\mathrm{CN}$

Following injections of FG into the left $\mathrm{CN}$, retrogradely labeled $\mathrm{CN}$-commissural cells were found in several areas throughout the contralateral CN, including the magnocellular region of $\mathrm{VCN}$, marginal region of VCN, SCC, the fusiform cell layer of the DCN, and deep DCN. CN-commissural neurons had polygonal, elongate, or round to oval somata. When both soma and dendrites were filled by BDA or FG, we found that most of the CN-commissural cells in the magnocellular region of the VCN were multipolar with varied sizes. The $\mathrm{CN}$-commissural cells in the marginal region were also multipolar and usually small in size. We were unable to identify the cell typing of the few labeled commissural neurons in the deep layer of DCN and VCN because their dendrites were not filled. These CN commissural cells usually had round/oval or elongated somata. Many CN commissural cells showed moderate-to-strong glycine immunoreactivity and weak GABA immunoreactivity. However, some retrogradely labeled $\mathrm{CN}$-commissural neurons did not colabel with either glycine or GABA (Figs. 3 and 4). Relative counts of cells with immunoreactivity to glycine or GABA are displayed in Table 1 . Thirty-seven percent of CN-commissural cells were immunonegative to both glycine and GABA, and they were either large or small in size.

The majority of glycine-negative and GABA-negative $\mathrm{CN}$ commissural neurons were located along the medial border of the $\mathrm{CN}$ (marginal region) or in the SCC (Fig. 5).
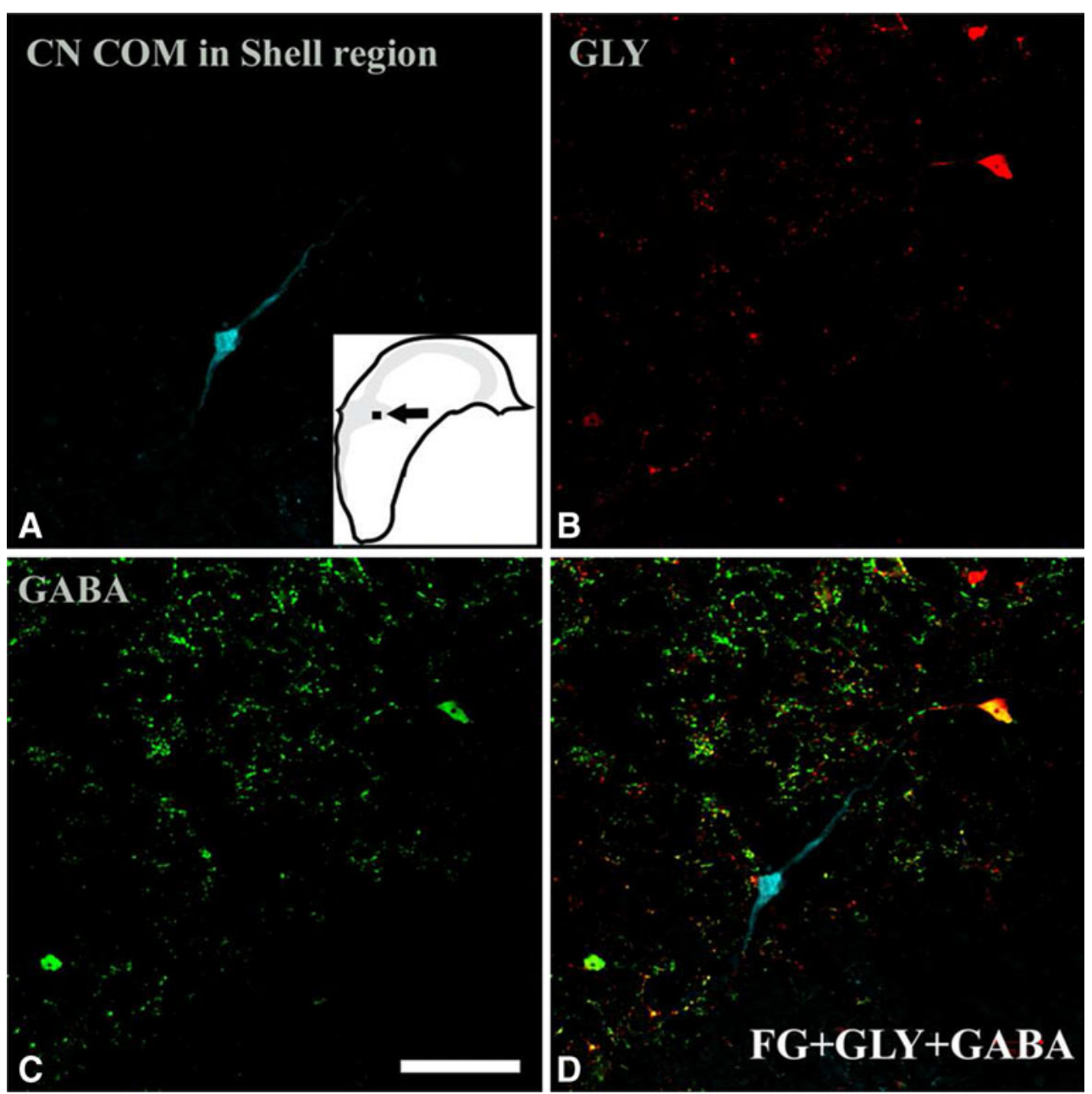

FIG. 3. Confocal images $(40 \times)$ of a retrogradely labeled CNcommissural neuron in the shell region of PVCN showing negative glycine immunoreactivity and negative GABA immunoreactivity. A The retrogradely labeled cell in the shell region following injections of $\mathrm{FG}$ in the contralateral $\mathrm{CN}$. The inset shows the location of the labeled cell. This cell shows both negative glycine immunoreactivity (B) and negative GABA immunoreactivity (C). Confocal multichannel image (overlap of A, B, and $\mathbf{C}$ ) shows no colocalization of FG with either glycine or GABA immunoreactivity (D). Scale bar $=50 \mu \mathrm{m}$. 

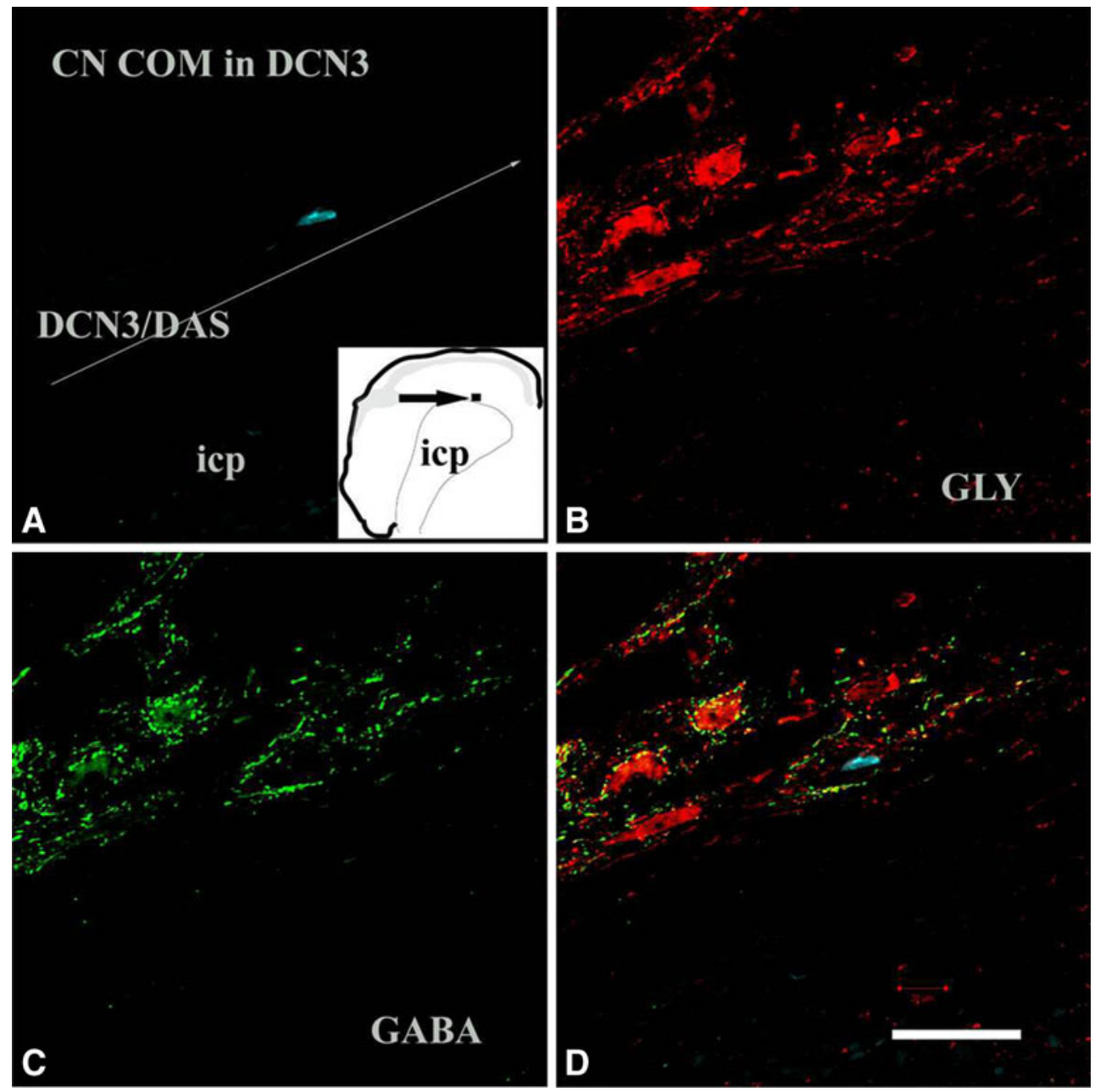

FIG. 4. Confocal images $(40 \times)$ of a retrogradely labeled $\mathrm{CN}$ commissural neuron in the DCN3 showing negative glycine immunoreactivity and negative GABA immunoreactivity. A The retrogradely labeled cell in the DCN3 following injections of FG in the contralateral $\mathrm{CN}$. The inset shows the location of the labeled cell. The straight line indicates the boundary between DCN and inferior

$\mathrm{CN}$-commissural cells that project to contralateral GCD mostly are located in marginal regions of the $\mathrm{CN}$

Since only CN commissural puncta in the GCD colabeled with VGLUT2, we further examined the

cerebellar peduncle (icp). This cell shows both negative glycine immunoreactivity (B) and negative GABA immunoreactivity (C). Confocal multichannel image (overlap of images of $\mathbf{A}, \mathbf{B}$, and $\mathbf{C}$ ) shows no colocalization of FG with either glycine or GABA immunoreactivity (D). Scale bar $=50 \mu \mathrm{m}$. DCN3 deep layer of DCN, DAS dorsal acoustic stria, ICP inferior cerebellar peduncle.

origin of these VGLUT2-positive CN-commissural neurons. Following a restricted injection of $\mathrm{BDA}$ into the GCD, the majority of labeled CN-commissural cells were found to be adjacent to and within the medial border of VCN, in the SCG region along the border between DCN and VCN (Fig. 6). These

TABLE 1

\begin{tabular}{|c|c|c|c|c|c|}
\hline \multicolumn{6}{|c|}{ GABA and GLY immunoreactivity of $\mathrm{CN}$-commissural cells } \\
\hline & \multicolumn{4}{|c|}{ GABA/Gly } & \multirow[b]{2}{*}{ Total } \\
\hline & $-1-$ & $+/-$ & $-/+$ & $+/+$ & \\
\hline Large cells & $76(18 \%)$ & $4(1 \%)$ & $36(8 \%)$ & $152(36 \%)$ & $268(63 \%)$ \\
\hline Small cells & $80(19 \%)$ & $8(2 \%)$ & $8(2 \%)$ & $60(14 \%)$ & $156(37 \%)$ \\
\hline Total & $156(37 \%)$ & $12(3 \%)$ & $44(10 \%)$ & $212(50 \%)$ & 424 \\
\hline
\end{tabular}

Summary of the number and percentage of $\mathrm{CN}$-commissural cells in one animal (see Fig. 5) that were double-labeled with GABA, glycine or GABA + glycine. Small cells are those with long axis $<16 \mu \mathrm{m}$, and large cells are those with long axis $>16 \mu \mathrm{m}$. Commissural cells that showed double-labeling with GABA or glycine are indicated by plus signs. Commissural cells that did not have any double-labeling with GABA or glycine are indicated by minus signs 


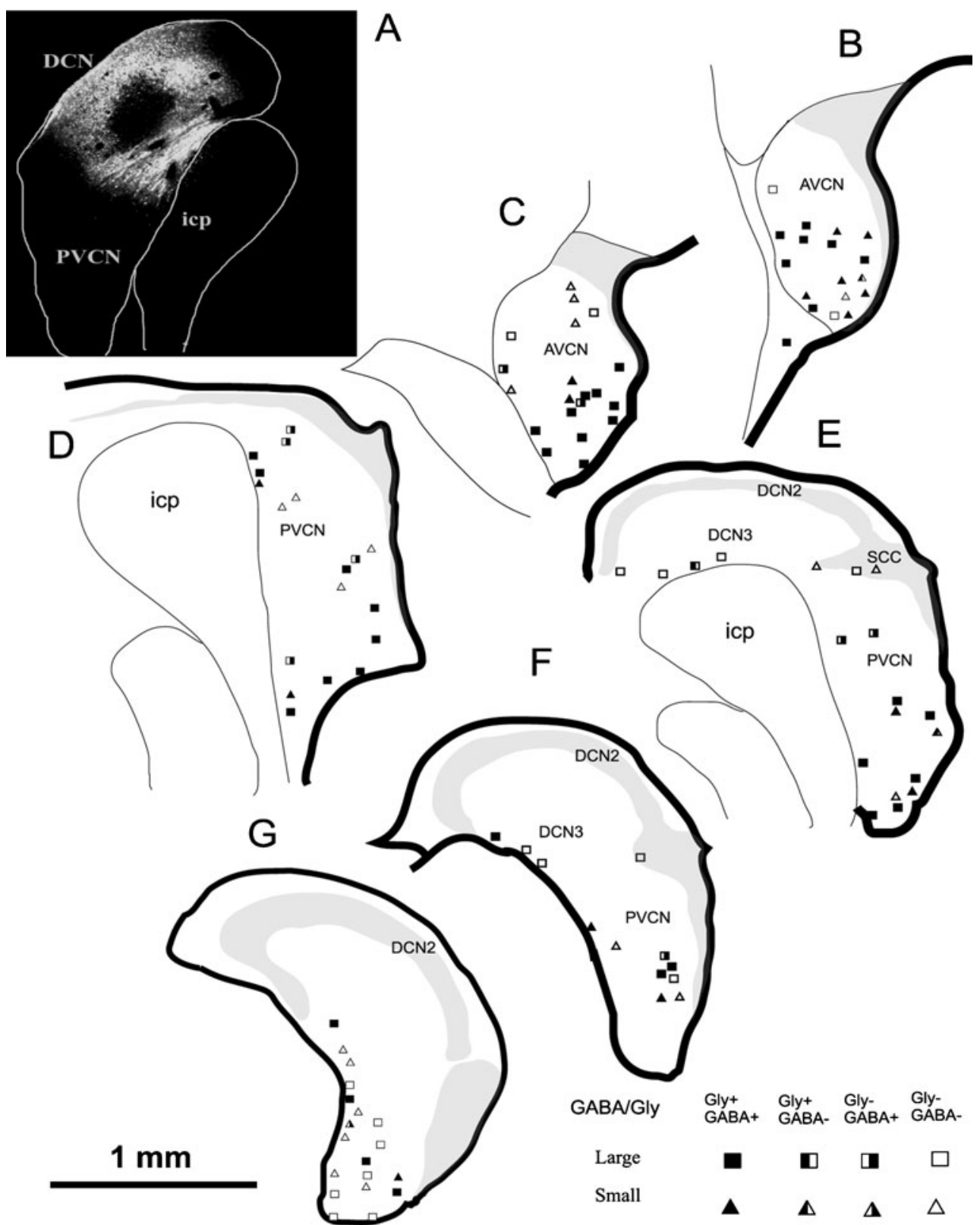

FIG. 5. Schematic drawing of $\mathrm{CN}$-commissural neurons with their glycine and GABA immunoreactivity. A The injection site in the contralateral CN. B-G Drawings of $\mathrm{CN}$ transverse sections that evenly extend from the caudal to rostral ends of the right $\mathrm{CN}$. The labeled $\mathrm{CN}$-commissural neurons were manually counted on every fourth section and mapped on the corresponding templates. Each symbol represents a labeled neuron. Symbols that represent

CN-commissural neurons had polygonal, elongate, or round to oval somata with varied sizes (Fig. 7). Labeled fibers in the right $\mathrm{CN}$ entered the $\mathrm{CN}$ through either the dorsal/intermediate acoustic stria or the trapezoid body. The distribution of these $\mathrm{CN}$-commissural cells is similar to that of the glycine negative/GABA-negative CN-commissural cells found in this study (Figs. 5 and 6). immunoreactivity to glycine or GABA are illustrated in the right lower corner. The majority of glycine negative (-) and GABA negative (-) $\mathrm{CN}$ commissural neurons are located in the medial and shell regions of VCN. Scale bar $=50 \mu \mathrm{m}$. DCN2 fusiform cell layer also containing granule cells and therefore part of GCD, DCN3 deep layer of DCN.

Some $\mathrm{CN}$-commissural cells receive VGLUT2-positive synaptic contacts

A number of retrogradely labeled commissural neurons received VGLUT2 immunoreactive terminals on their somata (Fig. 8). These neurons were located in the ventral part of either AVCN or PVCN, were medium to large in size, and had oval or round or 


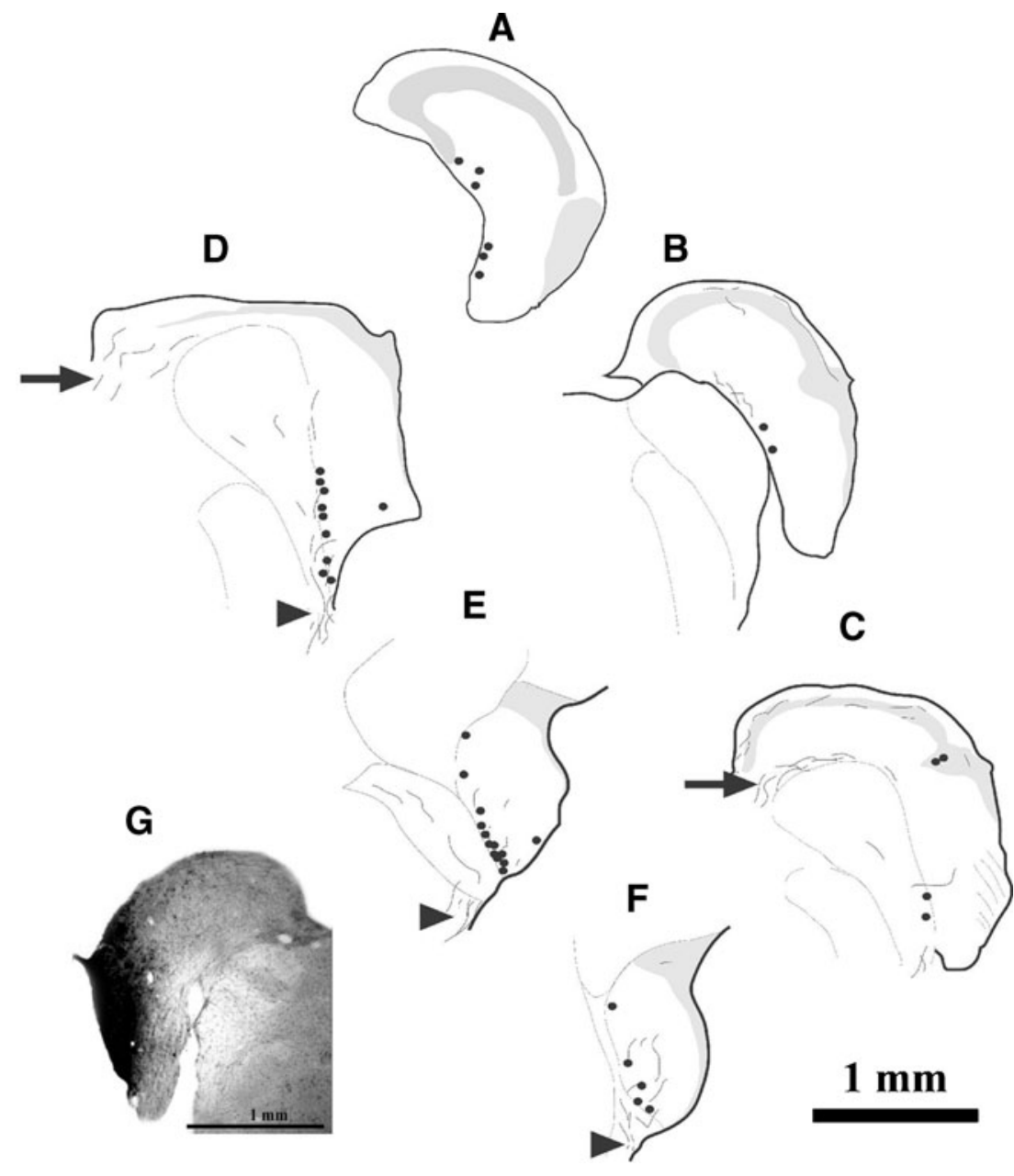

FIG. 6. Retrograde labeling in the $\mathrm{CN}$ following a restricted injection of $\mathrm{BDA}$ into the GCD of the contralateral VCN. A-F Drawings of rostral-to-caudal transverse sections across the right $\mathrm{CN}$. Each dot represents a labeled neuron. Lines indicate labeled fibers. The majority of labeled neurons were located adjacent to the medial borders of VCN; in the SCC; deep DCN and DCN/VCN border regions. Labeled fibers in the $\mathrm{CN}$ were located in either the dorsal/intermediate acoustic stria (arrows) or the trapezoid body (arrowheads). G The injection site in the left VCN. Scale bar $=1 \mathrm{~mm}$. elongate cell bodies that could not be further typed due to lack of dendritic filling. The VGLUT2-positive terminals were not double-labeled with FG and therefore probably do not arise from other commissural neurons.

\section{DISCUSSION}

This study provides compelling evidence that, in addition to inhibitory neurons, the CN-commissural projection is composed of excitatory cells. These excitatory $\mathrm{CN}$-commissural neurons are primarily found in the deep DCN, SCC, and marginal regions of VCN and project to the contralateral GCD.

The prevailing impression of a solely inhibitory commissural pathway probably arises from the abundant physiological data indicating that contralateral sound produces a predominant inhibitory response in the CN. Cant and Gaston (1982) found that the majority of $\mathrm{CN}$ commissural neurons are among the largest neurons in the $\mathrm{CN}$, which resemble the inhibitory type II multipolar cells. However, the ultrastructural and immunocytochemical characteristics of the
CN commissural pathway were first documented by Alibardi (1998, 2000), in which he showed that, contrary to the common notion, about two thirds of $\mathrm{CN}$ commissural neurons were immunonegative to GABA and Gly, with a preference for the putative excitatory commissural neurons in the VCN over the DCN. Here we found that $37 \%$ of $\mathrm{CN}$-commissural neurons were immunonegative to both glycine and GABA. The difference between the two studies may be due to the methods used to identify non-Gly/nonGABA neurons: we only included those that were unequivocally immunonegative to glycine and GABA as "excitatory".

Doucet et al. (2009) also reported that the CN commissural neurons were either large multipolar cells that resemble the glycinergic, type II cells or medium-sized multipolar cells that accounted for $69 \%$ of commissural cells and resembled type I cells, and are likely non-glycinergic or non-GABAergic. The present study supports their findings. The small- to medium-sized commissural cells account for $37 \%$ of the total labeled $\mathrm{CN}$-commissural cells and more than half of them (19\% in $37 \%$, Table 1) are nonglycinergic or non-GABAergic, whereas large cells 


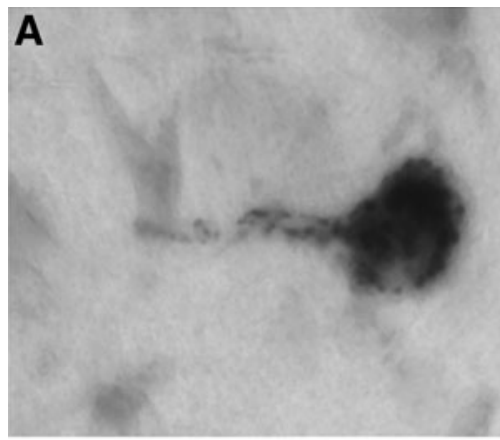

D
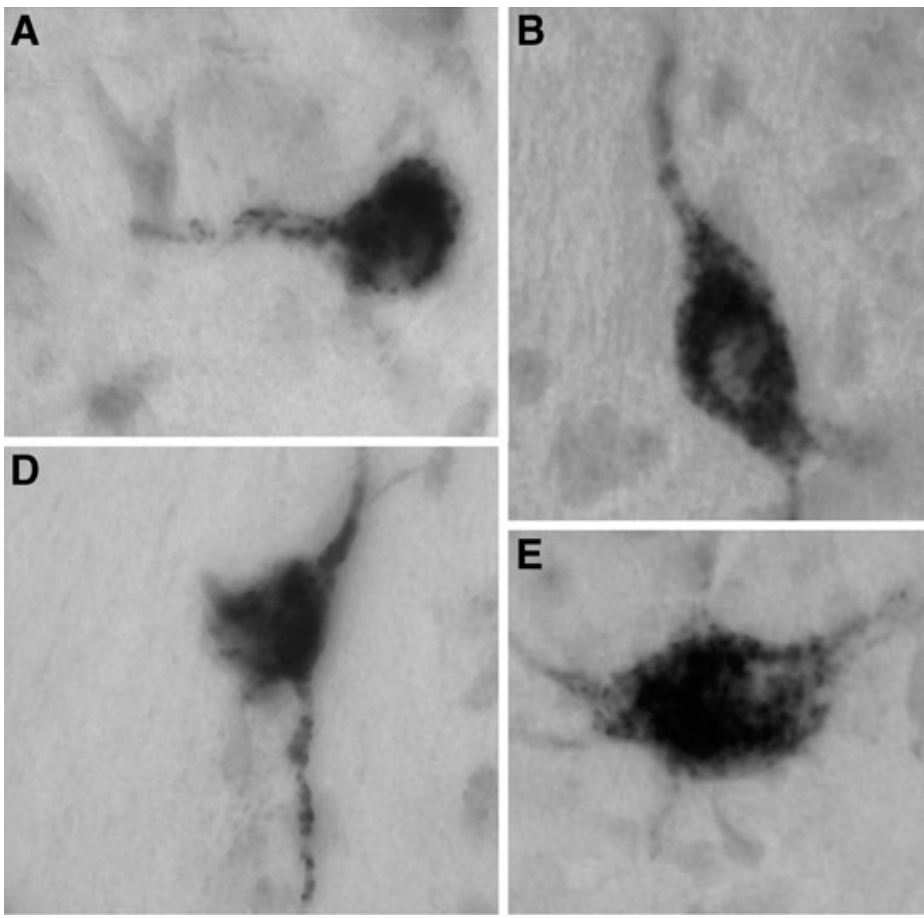
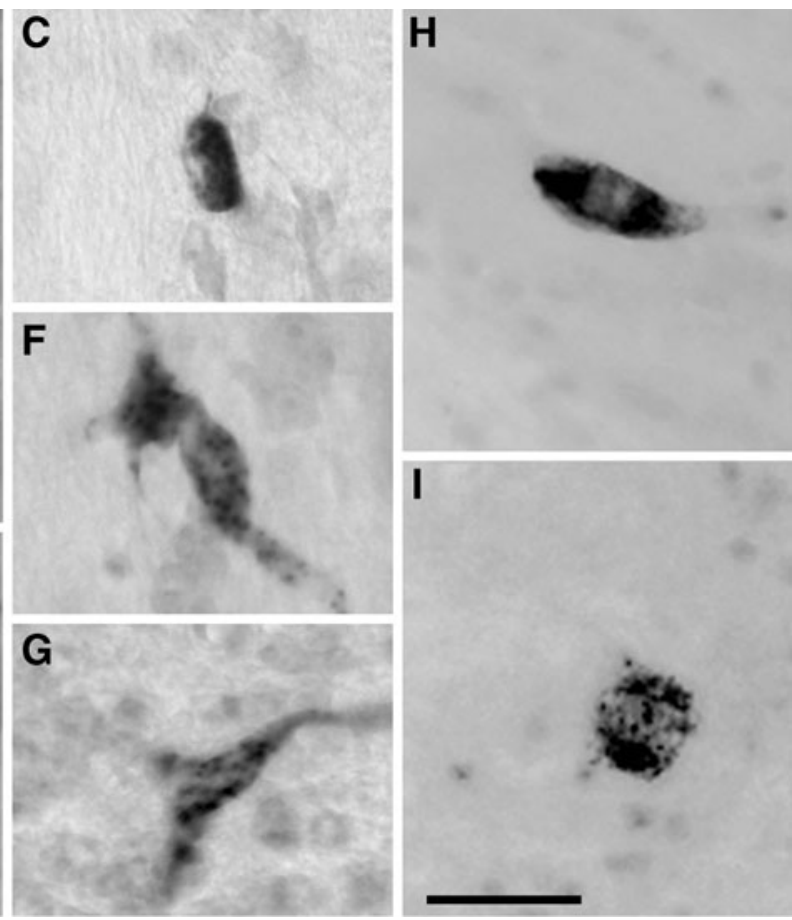

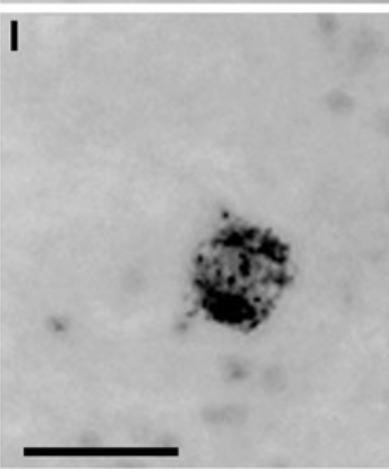

FIG. 7. Photomicrographs of labeled neurons in the $\mathrm{CN}$ following an injection of BDA into the contralateral GCD. The labeled $\mathrm{CN}$ commissural neurons in $\mathrm{VCN}$ had round-to-oval somata $(\mathbf{A}, \mathbf{B}, \mathbf{E}, \mathrm{F}$, I), polygonal $(\mathbf{D})$, or elongate somata $(\mathbf{C}, \mathbf{H})$, and had varied sizes.
The labeled commissural neurons in DCN had round or elongated somata and were located in DCN3 $(\mathbf{H}, \mathbf{I})$. The occasional labeled $\mathrm{CN}$-commissural cells in the SCC had polygonal somata $(\mathbf{C}, \mathbf{G})$. Scale bars $=50 \mu \mathrm{m}$. account for $63 \%$ of total labeled CN commissural cells and about $71 \%$ of those $(45 \%$ of the $63 \%$, Table 1 ) are immunopositive to glycine or/and GABA.

It is unclear why the anatomical studies do not agree well with the physiological observations of a predominant inhibitory $\mathrm{CN}$ commissural pathway in normal-hearing animals. Plausible explanations for this discrepancy include the following: (1) The present study showed that the putative excitatory commissural cells project to the GCD, a region in which it is difficult to place a recording electrode. The electrophysiological characteristics of small cells have only been documented in two studies (Ghoshal and Kim 1996, 1997), which showed that cells in this region are significantly different from those in the neural core groups of AVCN: they have low spontaneous firing rates, and show either no acoustic response or wide dynamic ranges to tones and noises. (2) The locations in the GCD, and morphology of the excitatory $\mathrm{CN}$-commissural terminal endings, as well as their immunoreactivity to VGLUT2 and not to VGLUT1, resemble that of non-auditory (somatosensory) projection neurons (Haenggeli et al. 2005; Wright and Ryugo 1996; Zhou et al. 2007; Zhou and Shore 2004). These non-auditory pathways can indirectly modulate DCN activity via parallel fibers, which can be either excitatory or inhibitory (Shore 2005; Davis et al. 1996). (3) The putative excitatory CN- commissural pathway may be dormant in normalhearing animals. The finding that $\mathrm{CN}$-commissural cells are surrounded by VGLUT2 immunoreactive terminals suggests that the CN-commissural pathway may be modulated largely by non-auditory inputs rather than by auditory nerve inputs, since auditory nerves use VGLUT1 to mediate glutamate release (Zhou et al. 2007). Further studies are needed to explore the notion that auditory nerve inputs may make preferential contacts with inhibitory CN-commissural neurons, whereas non-auditory inputs synapse with excitatory CN-commissural neurons.

Interestingly, following a restricted injection into the GCD, the retrogradely labeled CN-commissural neurons were found primarily in the medial border of $\mathrm{CN}$, which overlaps the locations of the GABA/Glynegative, excitatory $\mathrm{CN}$-commissural neurons, as well as some in the SCC, which were small multipolar neurons with either polyglonal or oval somata. Furthermore, the locations of these excitatory CNcommissural neurons correspond well with the locations of newly identified type I commissural neurons in the rat SCC, which show low-percentage apposition (i.e., percentage of the soma apposed by synaptic terminals) (Doucet et al. 2009). In that study, 69\% of CN-commissural neurons had type I axosomatic innervation, about a third of which were located in the SCC, also along the medial border of the VCN, consistent 

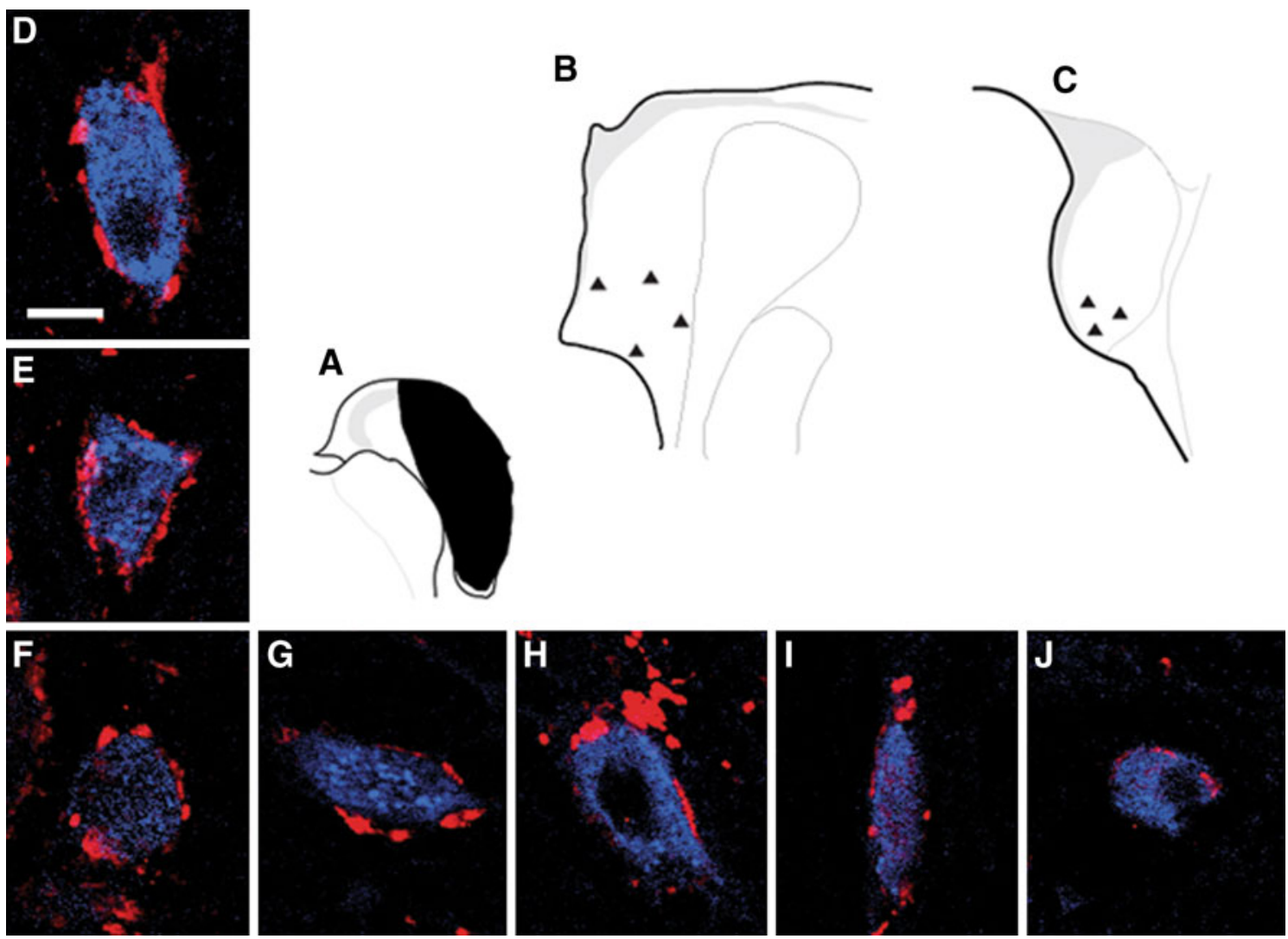

FIG. 8. Confocal images showing retrogradely labeled commissural neurons with somata surrounded by VGLUT2 immunoreactive terminals. A Schematic FG injection site in contralateral CN. B Schematic of the locations of commissural neurons in $\mathbf{G}-\mathbf{J}$. These neurons are located in the ventral part of AVCN, and have oval or round or elongate cell bodies with medium to large size. c Schematic of the locations of the commissural neurons in $\mathbf{D}-\mathbf{F}$. These neurons are located in the ventral part of PVCN, have oval or round large cell bodies, and are surrounded by positive VGLUT2 immunoreactive terminals. with the locations of excitatory $\mathrm{CN}$-commissural cells in the present study. The combined data from both these studies suggest that there is an excitatory neural connection between the marginal border of one $\mathrm{CN}$ and the contralateral GCD. Although other excitatory neurotransmitters such as acetylcholine may also be involved in the excitatory $\mathrm{CN}$-commissural pathway, the present study clearly demonstrated that this excitatory pathway, at least in part, is glutamatergic and uses VGLUT2 to mediate glutamate transport.

The inhibitory CN-commissural pathway has been implicated in encoding interaural timing and intensity differences important for sound localization (Cant and Gaston 1982; Doucet et al. 2009). In contrast, the paucity of excitatory responses to contralateral acoustic stimuli in normal-hearing animals suggests that this excitatory pathway is unlikely to play an important role in binaural interaction. However, removal of ipsilateral auditory inputs can dramatically enhance this "dormant" excitatory pathway from $4 \%$ to $30 \%$ (ipsilateral conductive hearing loss, Sumner et al. 2005) or $60 \%$ (sensorineural hearing loss, Bledsoe et al. 2009). These findings suggest that the $\mathrm{CN}$-commissural excitatory pathway may compensate for the diminished peripheral inputs to the ipsilateral $\mathrm{CN}$ after injury. This compensatory mechanism may help to maintain a balanced bilateral ascending flow of auditory information, essential for the optimal perception of acoustic characteristics such as timing and intensity.

Since the excitatory CN-commissural pathway resembles that of non-auditory (somatosensory) projection neurons (Dehmel et al. 2008; Haenggeli et al. 2005; Wright and Ryugo 1996; Zhou et al. 2007; Zhou and Shore 2004), it is likely to have a similar modulatory function as suggested for these non-auditory projections and may be associated with DCN synaptic plasticity under normal-hearing conditions (Tzounopoulos et al. 2004; Young et al. 1995; Zhou et al. 2007).

\section{ACKNOWLEDGEMENTS}

This study was supported by grants from the Royal National Institute for Deaf People, NIH P30 05188 and NIH R01 DC004825. We thank Sanford Bledsoe for helpful comments and suggestions. 


\section{REFERENCES}

Abercrombie M (1946) Estimation of nuclear population from microtome sections. Anat Rec 94:239-245

Alibardi L (1998) Ultrastructural and immunocytochemical characterization of commissural neurons in the ventral cochlear nucleus of the rat. Ann Anat 180(5):427-438

Alibardi L (2000) Cytology, synaptology and immunocytochemistry of commissural neurons and their putative axonal terminals in the dorsal cochlear nucleus of the. Anat Anz 182:207-220

AliBARDI L (2006) Review: cytological characteristics of commissural and tuberculoventral neurons in the rat dorsal cochlear nucleus. Hear Res 216-217:73-80

Allain AE, Bairi A, Meyrand P, Branchereau P (2006) Expression of the glycinergic system during the course of embryonic development in the mouse spinal cord and its co-localization with GABA immunoreactivity. J Comp Neurol 496:832-846

Babalian AL, Jacomme AV, Doucet JR, Ryugo DK, Rouiller EM (2002) Commissural glycinergic inhibition of bushy and stellate cells in the anteroventral cochlear nucleus. Neuroreport 13:555-558

Bledsoe SC Jr, Koehler S, Tucci Dl, Zhou J, Le Prell C, Shore SE (2009) Ventral cochlear nucleus responses to contralateral sound are mediated by commissural and olivocochlear pathways. J Neurophysiol 102:886-900

CAnt NB, Benson CG (2003) Parallel auditory pathways: projection patterns of the different neuronal populations in the dorsal and ventral cochlear nuclei. Brain Res Bull 60:457-474

Cant NB, Gaston KC (1982) Pathways connecting the right and left cochlear nuclei. J Comp Neurol 212:313-326

Crook J, Hendrickson A, Robinson FR (2006) Co-localization of glycine and gaba immunoreactivity in interneurons in Macaca monkey cerebellar cortex. Neuroscience 141:1951-1959

Dehmel S, Cui Y, Shore S (2008) Cross-modal interactions of auditory and somatic inputs in the brainstem and midbrain and their imbalance in tinnitus and deafness. Am J Audiol 17:193-209

Davis KA, Miller RL, Young ED (1996) Effects of somatosensory and parallel-fiber stimulation on neurons in dorsal cochlear nucleus. J Neurophysiol 76(5):3012-3024

Doucet JR, Rrugo DK (2006) Structural and functional classes of multipolar cells in the ventral cochlear nucleus. Anat Rec A Discov Mol Cell Evol Biol 288:331-344

Doucet JR, Lenihan NM, May BJ (2009) Commissural neurons in the rat ventral cochlear nucleus. J Assoc Res Otolaryngol 10:269-280

Fremeau RT Jr, Troyer MD, Pahner I, Nygaard GO, Tran CH, Reimer RJ, Bellocchio Ee, Fortin D, Storm-Mathisen J, Edwards RH (2001) The expression of vesicular glutamate transporters defines two classes of excitatory synapse. Neuron 31:247-260

Fremeau RT Jr, Burman J, Qureshi T, Tran CH, Proctor J, Johnson J, Zhang H, Sulzer D, Copenhagen DR, Storm-Mathisen J, Reimer RJ, Chaudhry FA, Edwards RH (2002) The identification of vesicular glutamate transporter 3 suggests novel modes of signaling by glutamate. Proc Natl Acad Sci USA 99:14488-14493

Gabellec MM, Panzanelli P, Sassoe-Pognetto M, Lledo PM (2007) Synapse-specific localization of vesicular glutamate transporters in the rat olfactory bulb. Eur J Neurosci 25:1373-1383

Gleich O, Vater M (1998) Postnatal development of GABA-and glycine-like immunoreactivity in the cochlear nucleus of the Mongolian gerbil (Meriones unguiculatus). Cell Tissue Res 293:207-225

Ghoshal S, Kiм DO (1996) Marginal shell of the anteroventral cochlear nucleus: acoustically weakly-driven and not-driven units in the unanesthetized decerebrate cat. Acta Otolaryngol 116: 280-283
Ghoshal S, KIm DO (1997) Marginal shell of the anteroventral cochlear nucleus: single-unit response properties in the unanesthetized decerebrate cat. J Neurophysiol 77:2083-2097

Hackney CM, Osen KK, Kolston J (1990) Anatomy of the cochlear nuclear complex of guinea pig. Anat Embryol (Berl) 182 (2):123-149

Haenggeli CA, Pongstaporn T, Doucet JR, Ryugo DK (2005) Projections from the spinal trigeminal nucleus to the cochlear nucleus in the rat. J Comp Neurol 484:191-205

InGHAM NJ, BLEECK S, WINTER IM (2006) Contralateral inhibitory and excitatory frequency response maps in the mammalian cochlear nucleus. Eur J Neurosci 24:2515-2529

Kaneko T, FujIXama F, Hioki H (2002) Immunohistochemical localization of candidates for vesicular glutamate transporters in the rat brain. J Comp Neurol 444:39-62

Kolston J, Osen KK, Hackney CM, Ottersen OP, Storm-Mathisen J (1992) An atlas of glycine- and GABA-like immunoreactivity and colocalization in the cochlear nuclear complex of the guinea pig. Anat Embryol (Berl) 186:443-465

Needham K, Paolini AG (2003) Fast inhibition underlies the transmission of auditory information between cochlear nuclei. $\mathrm{J}$ Neurosci 23:6357-6361

Needham K, Paolini AG (2007) The commissural pathway and cochlear nucleus bushy neurons: an in vivo intracellular investigation. Brain Res 1134:113-121

Ohshita N, Nakajo N, Takemura M (2004) Characteristics of the trigeminal depressor response in cats. J Neurosci Res 76:891-901

Pow DV, Wright LL, VANEY DI (1995) The immunocytochemical detection of amino-acid neurotransmitters in paraformaldehydefixed tissues. J Neurosci Methods 56:115-123

Schmued LC, Fallon JH (1986) Fluoro-Gold: a fluorescent retrograde axonal tracer with numerous unique properties. Brain Res 377:147-154

Schofield BR, CANT NB (1996) Origins and targets of commissural connections between the cochlear nuclei in guinea pigs. J Comp Neurol 375:128-146

SHORE SE (2005) Multisensory integration in the dorsal cochlear nucleus: unit responses to acoustic and trigeminal ganglion stimulation. Eur J Neurosci 21:3334-3348

Shore SE, Godfrey DA, Helfert RH, Altschuler RA, Bledsoe SC Jr (1992) Connections between the cochlear nuclei in guinea pig. Hear Res 62:16-26

Shore SE, Sumner CJ, Bledsoe SC, Lu J (2003) Effects of contralateral sound stimulation on unit activity of ventral cochlear nucleus neurons. Exp Brain Res 153:427-435

Smith PH, Rhode WS (1989) Structural and functional properties distinguish two types of multipolar cells in the ventral cochlear nucleus. J Comp Neurol 282:595-616

Smith PH, MAssie A, JoRIS PX (2005) Acoustic stria: anatomy of physiologically characterized cells and their axonal projection patterns. J Comp Neurol 482:349-371

Sumner CJ, Tucci DL, Shore SE (2005) Responses of ventral cochlear nucleus neurons to contralateral sound after conductive hearing loss. J Neurophysiol 94:4234-4243

Tzounopoulos T, Kim Y, Oertel D, Trussell LO (2004) Cell-specific, spike timing-dependent plasticities in the dorsal cochlear nucleus. Nat Neurosci 7(7):719-725

Weedman DL, Pongstaporn T, Ryugo DK (1996) Ultrastructural study of the granule cell domain of the cochlear nucleus in rats: mossy fiber endings and their targets. J Comp Neurol 369:345-360

Wright DD, Ryugo DK (1996) Mossy fiber projections from the cuneate nucleus to the cochlear nucleus in the rat. J Comp Neurol 365:159-172

Xin H, Yannazzo JA, Duncan RS, Gregg EV, Singh M, Koulen P (2007) A novel organotypic culture model of the postnatal 
mouse retina allows the study of glutamate-mediated excitotoxicity. J Neurosci Methods 159:35-42

Young ED, Nelken I, Conley RA (1995) Somatosensory effects on neurons in dorsal cochlear nucleus. J Neurophysiol 73(2):743-765

Zhou J, Shore S (2004) Projections from the trigeminal nuclear complex to the cochlear nuclei: a retrograde and anterog- rade tracing study in the guinea pig. J Neurosci Res 78:901907

Zhou J, Nannapaneni N, Shore S (2007) Vesicular glutamate transporters 1 and 2 are differentially associated with auditory nerve and spinal trigeminal inputs to the cochlear nucleus. J Comp Neurol 500:777-787 